\title{
EXPERIMENTAL STUDY OF AIR BUBBLES IN A SIMULATED CARDIOPULMONARY BYPASS SYSTEM WITH FLOW CONSTRICTION*广
}

\author{
I. TANASAWA \\ The University of Michigan. Ann Arbor. Mich. 48105, U.S.A.
}

\begin{abstract}
An experimental study is performed to examine the breaking of an air bubble in the flow passage of a simulated cardiopulmonary bypass system by means of a flow constriction. The purpose of the study is to discover a geometry of the flow constriction which is efficient in breaking air bubbles while providing the least resistance to the flow of blood. i.e. to develop a new device for the oxygenation of the blood in extracorporeal circulation.

Both plasma and water are used in the study. The use of plasma is to simulate the principal transport properties of the human blood and enable direct visualization of bubbles. Water is used for comparison with plasma to determine the influence of fluid properties on the breaking of bubbles. Several different shapes of flow constriction are tested. It is observed that as a result of rapid changes in the liquid pressure and bubble shape, an air bubble breaks into many bubbles at downstream from the flow constriction. The results are quantatively expressed by the number of baby bubbles vs. the flow rate.

It is disclosed that the flask-shape constriction is efficient in breaking air bubbles while providing ideal passage for the flow of blood. The number of baby bubbles is found to increase with an increase in the fluid viscosity.
\end{abstract}

\section{INTRODUCTION}

THE DYNAMIC behavior of a spherical gas bubble situated in an infinite liquid is governed by the so-called bubble dynamic equation. It can be expressed as (Yang et al.. 1966).

$$
\ddot{R} R+\frac{3}{2} \dot{R}^{2}=\frac{p_{g}-p_{x}}{\rho}-\frac{2 \sigma}{\rho R}-4 \frac{\mu \dot{R}}{\rho R}
$$

where $R$ is the bubble radius, $\dot{R}$ and $\ddot{R}$ indicate the first-and second-order derivatives of $R$ with respect to time, $p_{g}$ is the pressure of gas or vapor confined in the bubble, $p_{x}$ is the pressure of the liquid at a great distance from the bubble, $\sigma$ is the surface tension, $\rho$ is the liquid density and $\mu$ is the liquid viscosity.

The two terms on the left side of equation (1) measure inertia force induced by the motion of the bubble, while the terms on the right side denote the pressure force, surface tension force and viscous force, respectively. An examination of equation (1) reveals that a bubble will grow in size when the liquid pressure $p_{x}$ decreases and vise versa. Although the equation applied only to a stationary bubble. it resembles the expression for the first approximation of the dynamic behavior of a moving bubble (Yeh et al., 1968), except the latter equation includes more terms expressing the effects of the liquid velocity, the relative velocity between the liquid and the bubble. the liquid acceleration, etc. In the present study concerning with blood flow in a cardiopulmonary bypass system, these effects are small. Hence, equation (1) can be employed to describe the bubble dynamics in the bypass system. This approximation will be verified later by experiments.

Consider the case in which a constriction is placed in a flow passage which is otherwise

*Received 19 January 1970.

tThe work reported was partially supported by the National Heart Institute and the Michigan Heart Association. The paper will be presented at 1970 SESA Spring Meeting to be heid in Huntsville, Alabama on May 19-22.

$\ddagger$ Michigan Heart Association Fellow from the University of Tokyo, Japan.

\$Graduate Student of Mechanical Engineering.

"Associate Professor of Mechanical Engineering.

TStudent of Mechanical Engineering. 
constant in cross section. In order to satisfy the continuity principle, the liquid is speeded up as it flows through the constriction. The flow velocity decreases as the liquid leaves the constriction and approaches a value at downstream. Vortices or turbulent wakes may be created in the downstream near the constriction. According to the Bernoulli's theorem, hydrodynamic pressure is decreased as the liquid flow is accelerated in the constriction. It reaches a minimum somewhere in the constriction where flow velocity is a maximum. The local liquid pressure increases downstream from the constriction and approaches a value when constant velocity is developed.

For a gas bubble riding on a stream through the constriction, it experiences first growing then shrinking processes due to the variation in the pressure of the surrounding liquid. However, because of the shape of the constriction and the application of asymmetric pressure forces, etc. a nearly spherical bubble (when its size is less than $5 \mathrm{~mm}$ in dia.) may be distorted even before it enters the constriction. The asymmetry in bubble shape may be further amplified during its passing through the constriction and the bubble eventually breaks up into pieces at the exit from the constriction or downstream in the vortex or turbulent wake region.

After a bubble breaks up into pieces, the baby bubbles which are smaller than the mother bubble become spherical or nearly spherical due to the action of surface tension force. The pressure difference $\left(p_{g}-p_{x}\right)$ is related to surface tension by

$$
p_{g}-p_{x}=\frac{2 \sigma}{R}
$$

which can be obtained from the balance of forces acting on the bubble surface. Furthermore, since the baby bubbles are much smaller than the mother bubble, equation (2) indicates that the pressure difference for the baby bubbles will be much larger than that for the mother bubble.
Small bubbles are more favorable for absorption of gas into liquid than a larger one for the following reasons: (1) the total gas-liquid interfacial area for mass transfer is increased. (2) the surface tension force $2 \sigma / R$ is increased as the size of a bubble is reduced. This increase in surface tension force results in the acceleration of bubble collapse as indicated in equation (1). (3) The rate of gas diffusion into the surrounding liquid is increased. As was mentioned in the previous paragraph, the pressure difference $p_{g}-p_{\infty}$ or the gas pressure $p_{g}$ itself may be considerably increased through the breaking process. According to the Henry's law, the solubility of gas in liquid is proportional to the gas pressure $p_{g}$. Hence, the rate of gas diffusion into the liquid may be enhanced, resulting in faster collapse of gas bubble by mass diffusion. This is particularly important to gas bubbles in extracorporeally oxygenated blood. Since most of the oxygen in blood is stored in hemoglobin, the absorption of oxygen from bubbles depends on the diffusion of oxygen out of the bubble. When the diffusion rate is enhanced due to an increase in $\left(p_{g}-p_{\infty}\right)$, it is expected that oxygen bubbles may collapse faster and less oxygen bubbles will enter human body to cause gas embolism (Chan et al., 1969).

The purpose of the present study is to discover the shape of a flow constriction in a stimulated cardiopulmonary bypass system which is efficient in breaking gas bubbles, yet provides the least resistance to the flow of blood. The motivation is to develop a new device to oxygenate the blood in extracorporeal circulation. In the experiments, air bubbles are injected into the circulating stream of plasma or water. The number of baby bubbles resulting from the splitting of a mother bubble is considered a measure of the effectiveness of the flow constraint.

\section{DESCRIPTION OF APPARATUS}

The test apparatus used for the study is shown in Fig. 1. It consists of a Sarns Model 3500 roller pump, a heat exchanger, a blood 


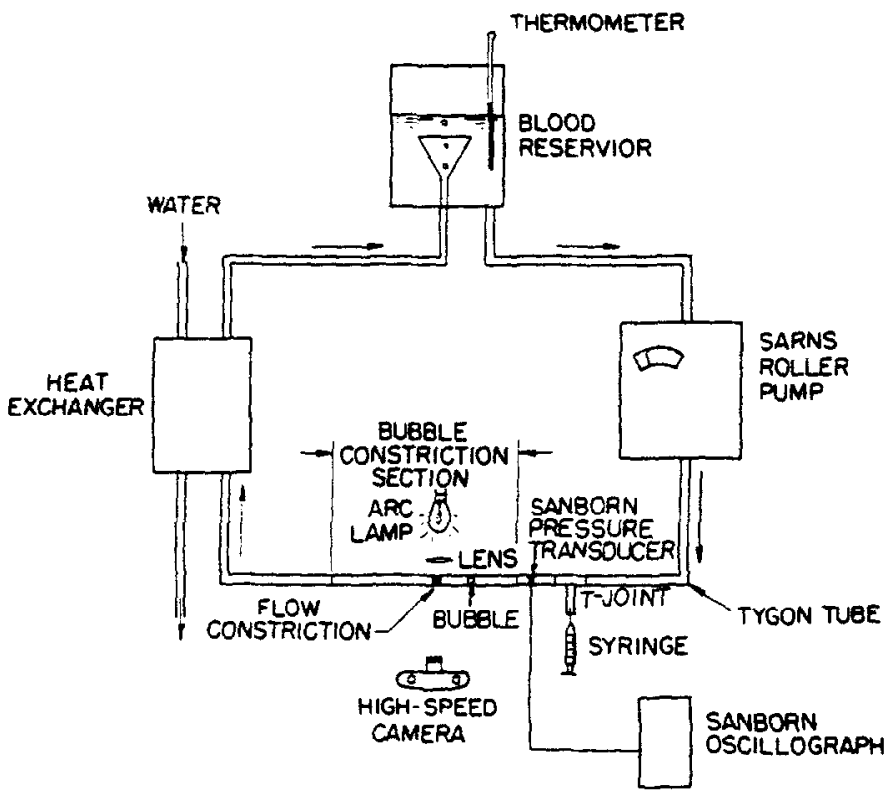

Fig. 1. Experimental apparatus.

reservoir and a bubble observation section. Both water and plasma were used in vitro experiments. The use of plasma is to simulate the principal transport properties of the human blood and enable direct visualization of moving gas bubbles. The influence of fluid viscosity and surface tension on the breaking of gas bubbles can be examined by comparing the results for water and plasma.

The roller-type pump provides a pulsating flow through the system. The arterial and venous lines consisted of tygon tubing of $\frac{3}{8}$ in. in dia. By adjusting the pump, flow rates can be varied from zero to $62 \mathrm{cc} / \mathrm{sec}$ in the $\frac{3}{8}$ in. dia. tubing. The fluid enters the blood reservoir through the stem of a funnel fixed to the base of the reservoir. The funnel serves to vent the gas bubbles contained in the fluid. Hence the reservoir functions also as a bubble trap. The fluid was pumped from the blood reservoir by the pump and returned to the blood reservoir through the bubble-behavior observation section and the heat exchanger. The bubblebehavior observation section consisted of a $25 \mathrm{~cm}$ long, $10 \mathrm{~mm}$ i.d. glass tubing. A flow constriction cut from a thin core disc of approximately $3 \mathrm{~mm}$ thick was placed inside the glass tubing. Several flow constraints of different geometry were tested. These included U-shape, V-shape, U-over- $\mathrm{I}$ shape, arc shape and flask shape. An air bubble was injected into the upstream end of the bubbleobservation section by means of a hyperdermic syringe. As the bubble flowed through the constriction its shape and size changed simultaneously. These changes in the bubble size and shape were recorded by Beckman-Whitley high-speed camera $(25,000 \mathrm{fps}$ framing) with the aid of a $100 \mathrm{~W}$ concentrated arc lamp and an achromatic lens. The pulsating liquid pressure at $10 \mathrm{~cm}$ upstream from the flow constriction was read by Sanborn model 267BC pressure transducer and recorded in a Sanborn model 150 oscillograph. A thermometer was installed at the reservoir to measure the fluid temperature.

\section{TEST PROCEDURE}

The pump was set at a desired flow rate for a sufficient time until all gas bubbles trapped in the fluid were vented through the funnel in the blood reservoir and a desired steady tem- 
perature was reached in the liquid. After the flow rate and liquid temperature were recorded, the pump was stopped, leaving the setting of the flow rate untouched. An air bubble was then injected into the simulated bypass system by the syringe at $25 \mathrm{~cm}$ upstream from the flow constriction.

The pump was then set in operation again at the previously set flow rate. The flow rate was established before the air bubble reached the constraint. The pulsating liquid pressure picked up by the pressure transducer at downstream from the constriction was recorded on the Sanborn oscillograph. The high-speed camera focused on the bubble observation section photographed the bubble as it entered and left the flow constriction. After the air bubble passed through the flow constraint and split into small baby bubbles downstream from the constraint, the pump was stopped again. The number of the baby bubbles were recorded.

\section{EXPERIMENTAL RESULTS}

Figure 2 shows a typical oscillographic record of fluid pressure variation at $10 \mathrm{~cm}$ upstream from the flow constriction detected by the Sanborn pressure transducer. The test run corresponded to a pulsating flow with the

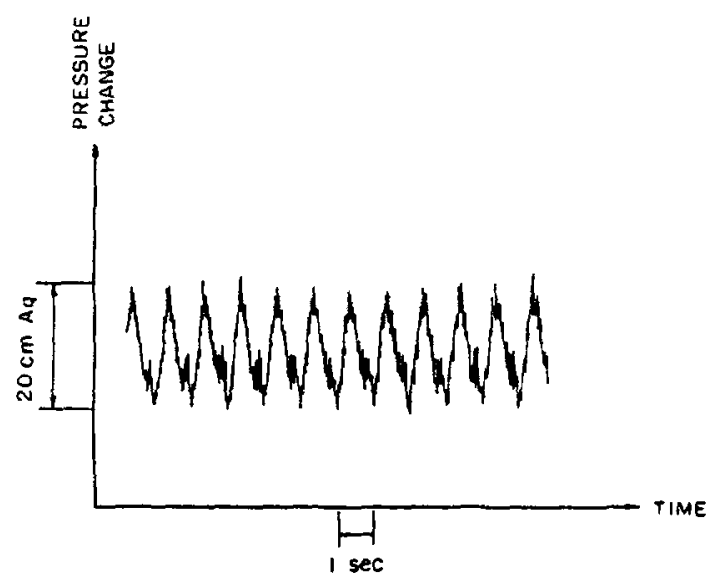

Fig. 2. Oscillographic record of pulsating liquid pressure in the simulated cardiopulmonary bypass system at frequency of 60 pulses $/ \mathrm{sec}$ and amplitude of $10 \mathrm{~cm}$ Aq. frequency of 60 pulses/sec (equivalent to the mean flow velocity of $10 \mathrm{~cm} / \mathrm{sec}$ in a $10 \mathrm{~mm}$ i.d. tube) and the amplitude of $10.0 \mathrm{~cm} \mathrm{Aq}$.

High-speed photographs of the bubbles were taken at the observation section of the system. When a flow constriction was installed in the section, the camera was focused on the constriction. Behind the tube, there is an achromatic lens through which the light from the concentrated arc lamp is focused on the constriction. Photographs were taken on $35 \mathrm{~mm}$ Kodak tri-X pan film. The film speed was set at $200 \mathrm{fps}$. Figure 3 shows typical photographs of an air bubble with initial size of $1.5 \mathrm{~mm}$ dia. in water. flowing through the bubble observation section without flow constriction. The sequence of the films were taken over a very short period of time, which is equivalent to approximately a point on the curve in Fig. 2. An examination of the photographs with the aid of a film projector has indicated that during this time period the changes in the bubble size and shape are very small. This implies that the effects of translatory motion of the bubble on its dynamic behavior, i.e. the changes in the bubble shape and size, are indeed negligible in the tested flow range. It is estimated using equation (1) the bubble size varies less than one per cent from its mean diameter over the pulsating pressure with the amplitude of 10.0 $\mathrm{cm} \mathrm{Aq}$. When the flow velocity is increased to $75 \mathrm{~cm} / \mathrm{sec}$, the maximum attainable flow rate in the Sarns roller type pump, the maximum change in the bubble size is estimated to be 5 per cent.

Figure 4 shows the photographs of an air bubble with the initial size of $1.5 \mathrm{~mm}$ dia. in the pulsating stream of water through a $15^{\circ}$ $V$-shape flow constriction. The pulsation was at the frequency of 60 pulses $/ \mathrm{sec}$ and the amplitude of $10 \mathrm{~cm}$ Aq. The frame speed was set at $900 / \mathrm{sec}$. The first five frames show the bubble at upstream from the flow constriction. The sixth frame shows part of the bubble has passed through the constriction while the rest of the bubble is still in the constriction. The seventh frame shows some of the baby bubbles near the 


\section{$11+1|1| 1|1|$}

23

Xo

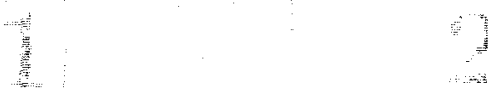

Fig. 3. Photographs of an air bubble with initial size of $1.5 \mathrm{~mm}$ dia. in a pulsating flow with frequency of 60 pulses $\mathrm{sec}$ and amplitude of $10 \mathrm{~cm} \mathrm{Aq.} 200 \mathrm{fps}$.
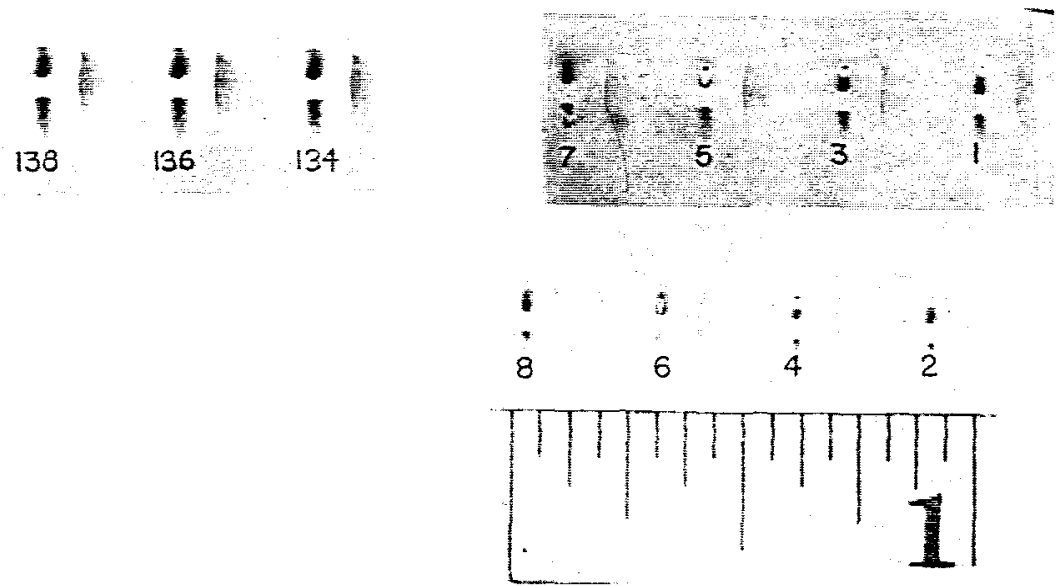

Fig. 4. Photographs of an air bubble with initial size of $1.5 \mathrm{~mm}$ dia. in a pulsating flow with frequency of 60 pulses/sec and amplitude of $10 \mathrm{~cm} \mathrm{Aq}$ through $15^{\circ} \mathrm{V}$. shape constriction, 900 fps. 
tube surface facing the camera. The majority of these baby bubbles are mixed in the bulk of the wake region and cannot be seen by the camera. In the following 126 frames, different numbers of baby bubbles are shown in the photographs, indicating that vigorous eddy motion is taking place in the wake region. The last photograph in Fig. 4 corresponds to the 138 th frame in which a few baby bubbles near the tube surface facing the camera are shown.

Experimental results were correlated for the number of smaller baby bubbles as a function of flow rate. Several different shapes of flow constraint were employed. The shape was selected for the purpose of identifying the geometry which is most effective in breaking a gas bubble. The results were presented in Figs. 5 through 9 . The locus of the minimum numbers of the broken pieces is represented by a solid line for plasma and by a broken line for water.

Figure 5 illustrates the results of both plasma and water for the arc-shape flow constriction. Strong eddy motion is seen in the

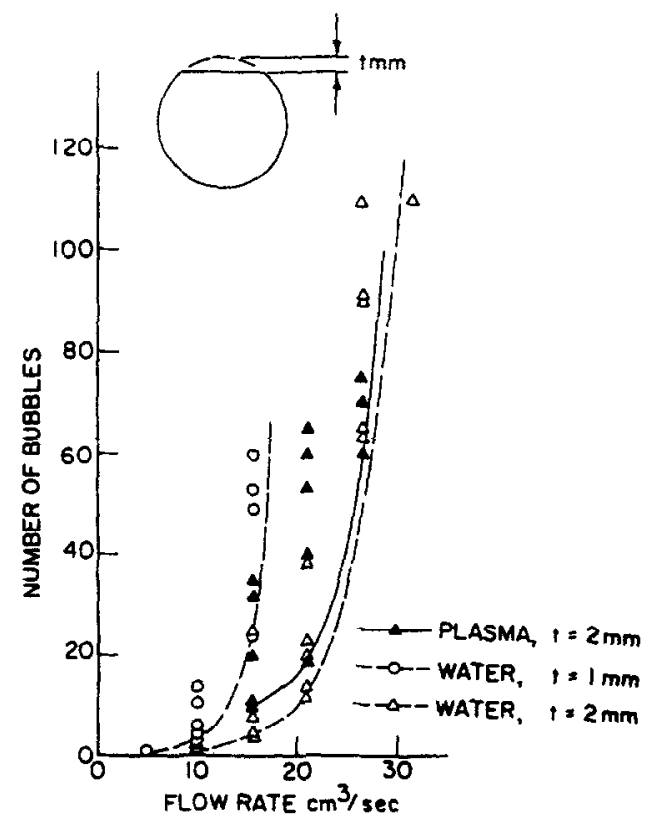

Fig. 5 . Number of baby bubbles vs. flow rate, for a $3 \mathrm{~mm}$ dia. bubble passing through arc-shape flow constrictions.

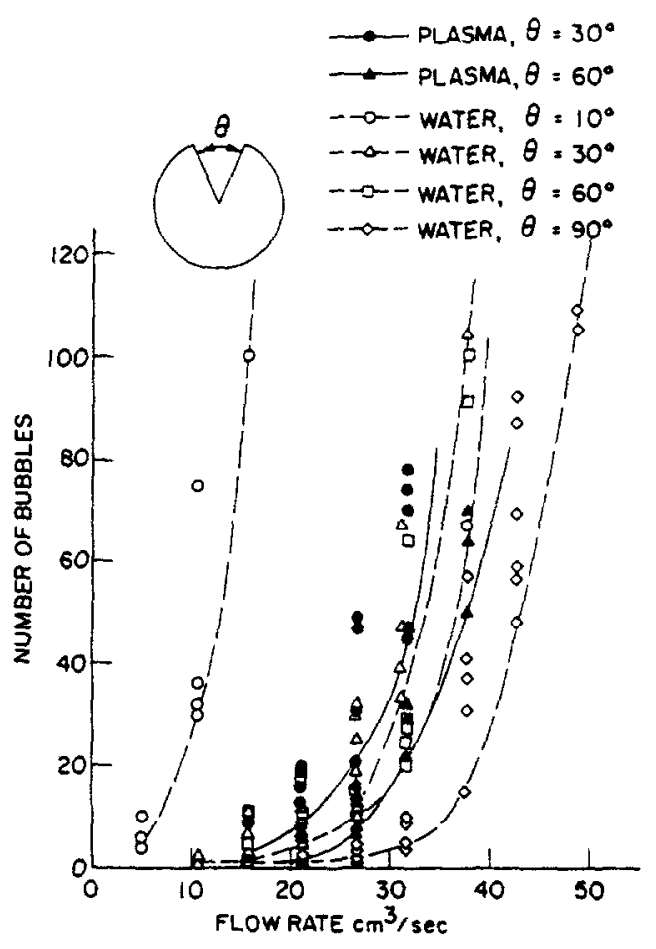

Fig. 6. Number of baby bubbles vs. flow rate. for a $3 \mathrm{~mm}$ dia. bubble passing through $\mathrm{V}$-shape flow constrictions.

downstream region where the bubble severely distorted by the opening of the constriction splits into small pieces. Since the pressure change across the constriction is inversely proportional to the square of the flow crosssectional area of the constraint, an increase in $t$ from $l$ to $2 \mathrm{~mm}$ results in a significant decrease in the number of small bubbles. The experimental results for $V$-shape flow constrictions with $\theta=10^{\circ}, 30^{\circ}, 60^{\circ}$ and $90^{\circ}$ are depicted in Fig. 6. The curves shift toward high flow rates as the angle $\theta$ increases. The results for U-shape constrictions are illustrated in Fig. 7. The comparison of Figs. 6 and 7 reveals that an air bubble is more distorted in shape as it goes through a U-shape constriction than a $V$-shape one and that more rigorous eddy motion has ta'en place downstream of the $U$ shape constriction than $V$-shape one.

In both Figs. 8 and 9 , the number of baby bubbles and the average radius of the largest 


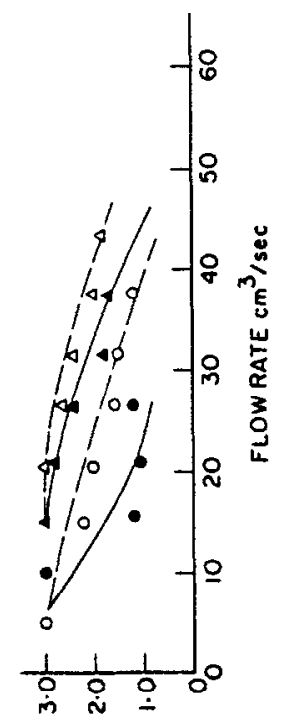

wा $4 \exists \times \forall 348$ \&31 t S3า JO SnIOVy $3904 \exists \wedge \theta$
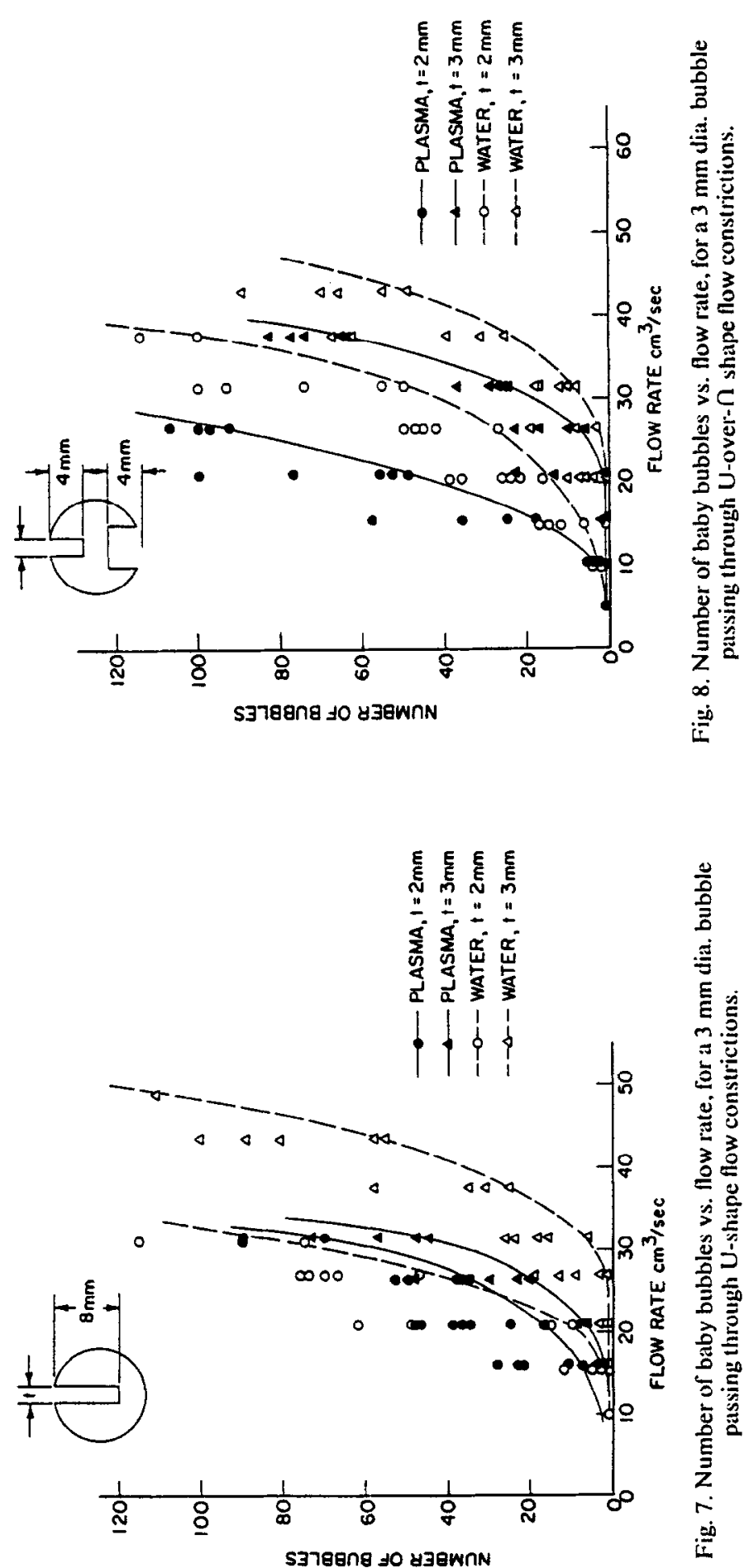

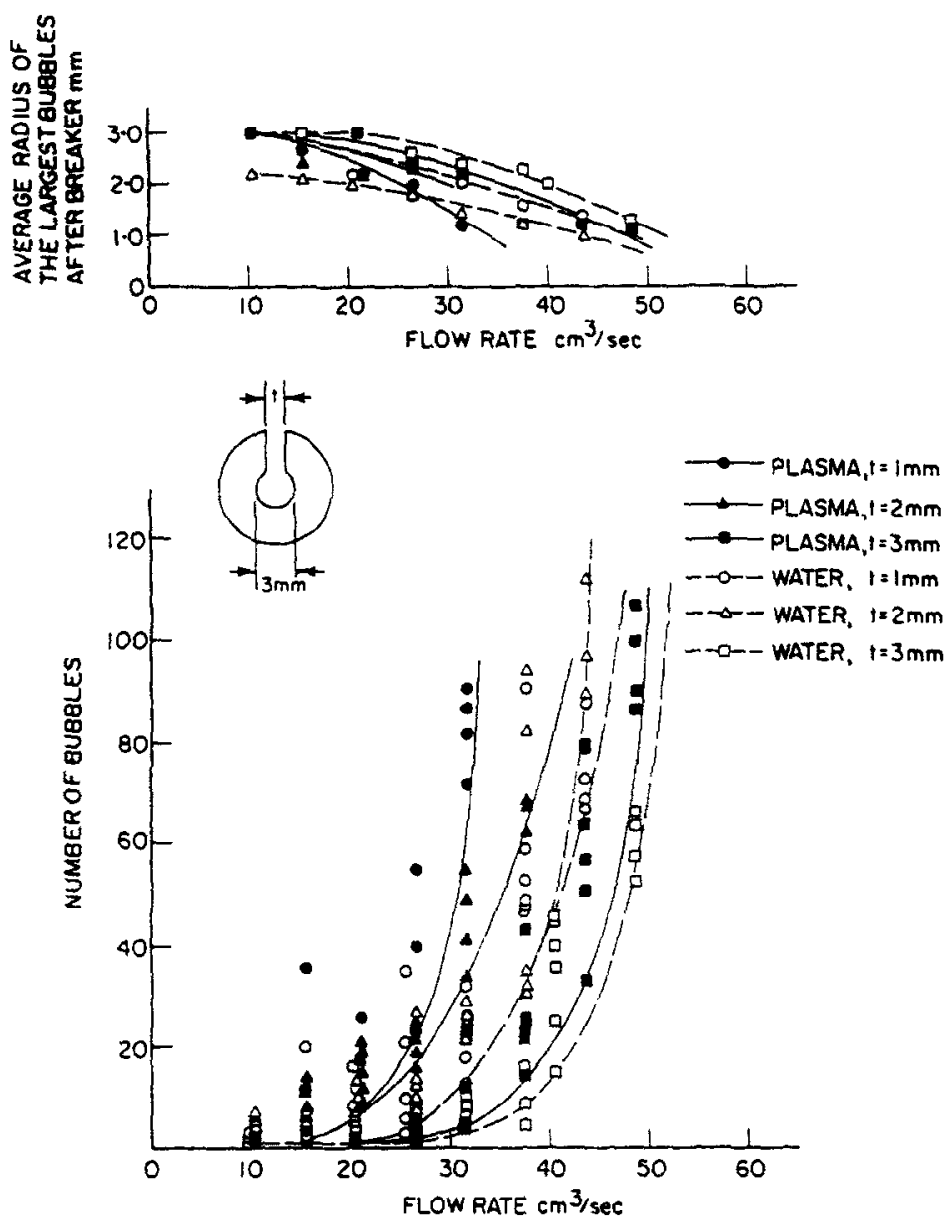

Fig. 9. Number of baby bubbles vs. flow rate, for a $3 \mathrm{~mm}$ dia. bubble passing through flask-shape flow constrictions.

baby bubbles produced in the wake region are plotted against the flow rate. The average radius of the largest baby bubble is obtained by averaging the radius of the largest bubble which appeared in each of several tests conducted for each flow rate. Although the U-over- $\cap$ shape is slightly favorable over the flask shape in producing baby bubbles, the latter is convenient for the flow of blood which is characterized by the Bingham-plastic type profile (with uniform velocity profile at the center portion of a flow cross section).

It is illustrated in all of Figs. 5 through 9 that more small bubbles are produced from breaking a single visual one in plasma than in water. This can be interpreted as follows. When a bubble surface is subject to a rapid change of an asymmetric pressure force exerted by the surtounding liquid, the surface tends to restore its shape through the action of surface tension. Since the surface tensions of the air-plasma and air-water systems are approximately the same, it must be the liquid viscosity which plays a role in the restoration of bubble shape. Hence, one concludes that a distorted bubble is less capable to restore to a spherical shape in high viscosity liquid (plasma has viscosity higher than water) and the coalescence of small bubbles in the wake region is less frequent. 


\section{CONCLUSIONS}

(1) The influence of pulsating pressure on the size and shape of an air bubble in the cardiopulmonary bypass system is insignificant in the range of the flow rates being investigated. Therefore the bubble may be considered under quasi-steady state.

(2) In the presence of a constriction in the flow passage, a bubble may break into many small pieces. The number of the resulting small bubbles depends on the shape of the constraint and the flow rate. The flask-shape constraint is considered the most favorable geometry as it is efficient to break bubbles and provide the least resistance to the flow of blood.

(3) In a high viscous fluid a bubble can be broken into more smaller bubbles through a constriction and the coalescence of the small bubbles is less.
(4) The mechanism of breaking a bubble into smaller bubbles through a constriction can be applied to develop a new type of oxygenator. The effectiveness and compactness of the new device will depend on the geometry of the constriction and its arrangement.

Acknowledgement-The work reported was partially supported by grants from the Michigan Heart Association and the National Institute of Health to which the authors wish to express their appreciation.

\section{REFERENCES}

Chan. K. S. and Yang. W. J. (1969) Survey of literature related to the problems of gas embolism in human body. J. Biomechanics 2. 299-312.

Yang. W. J. and Yeh. H. C. (1966) Theoretical study of bubble dynamics in purely viscous fluids. A.l.Ch.E. Jl 12. 927-931.

Yeh. H. C. and Yang. W. J. (1968 Dynamics of bubbles moving in liquids with pressure gradients. J. appl. Phys. 39. 3156-3165. 